Shoesmith, Emily ORCID:

https://orcid.org/0000-0002-2420-4919, Charura, Divine and Surr, Claire ORCID: https://orcid.org/0000-0002-4312-6661 (2020) Acceptability and feasibility study of a six-week person-centred, therapeutic visual art intervention for people with dementia. Arts \& Health. pp. 1-19.

Downloaded from: http://ray.yorksj.ac.uk/id/eprint/4713/

The version presented here may differ from the published version or version of record. If you intend to cite from the work you are advised to consult the publisher's version: http://dx.doi.org/10.1080/17533015.2020.1802607

Research at York St John (RaY) is an institutional repository. It supports the principles of open access by making the research outputs of the University available in digital form. Copyright of the items stored in RaY reside with the authors and/or other copyright owners. Users may access full text items free of charge, and may download a copy for private study or non-commercial research. For further reuse terms, see licence terms governing individual outputs. Institutional Repository Policy Statement

\title{
RaY
}

Research at the University of York St John

For more information please contact RaY at ray@yorksj.ac.uk 


\section{Acceptability and feasibility study of a six-week person-centred, therapeutic visual art intervention for people with dementia.}

\section{Abstract}

Background: There has been increasing interest in dementia, the arts and creativity across different disciplines in recent years, with previous literature illustrating the benefits of visual arts for people with dementia. Method: A mixed-methods, quasi-experimental, pre/post design to assess the feasibility, acceptability and preliminary efficacy of a newly developed therapeutic, person-centred visual art intervention for people with dementia attending a day care centre or residing in an assisted living facility. Results: Five themes were identified from the interviews. Two themes reflected the feasibility/acceptability and the perceived impacts of the intervention, and three themes represented perceived successful elements: participant choice, socialisation and mentally stimulating activities. The quantitative data tentatively indicated enhanced social functioning and quality of life scores post-intervention. Conclusion: These findings indicate that engagement with visual art is effective for people with dementia, and taking into account the factors that impact on feasibility and acceptability will promote future robust evaluation.

3 - 5 key words: dementia, creativity, visual arts, intervention evaluation, person-centred

Word Count: 7233 


\section{Introduction}

There has been an increasing interest in research focusing on dementia and the arts (Palmiero, Di Glacomo, \& Passafiume, 2012), especially as creative expression in artistic activities has been found to be an important way for people with dementia to express and access emotions despite cognitive impairment (Zeilig, Killick, \& Fox, 2014). Creative interventions that utilise art, music, dance and drama can be effective psychosocial approaches for people with dementia (de Medeiros \& Basting, 2013). In particular, research has illustrated the perceived positive impact of visual art interventions for people with dementia, such as improvements in wellbeing, quality of life (QoL) and communication (Richards et al., 2018; Windle et al., 2018). Furthermore, it is widely accepted that arts-based approaches are important in a variety of ways for ameliorating the lives of people with dementia (Creative Health, APPG, 2017).

Thus, the comprehensive exploration of the therapeutic potential of art-based interventions is becoming increasingly important (Schall, Tesky, Adams, \& Pantel, 2018). Within visual arts, a number of programmes have been implemented to provide opportunities for people with dementia to participate in the creation of artwork using a range of visual arts media. The focus of the activities is often on the positive influence of engagement and participation (e.g. Sauer, Fopma-Loy, Kinney, \& Lokon, 2016). This differs from art therapy, whereby the arts serve as a language to express and reveal an individual's thoughts and emotions (Brillantes-Evangelista, 2013). Mangione (2018) reported that art therapists tended to discuss art as instrumentally valuable, as the means to a specifically therapeutic end. Conversely, for art-making facilitators, the arts have intrinsic value and their responsibility is to promote participants' appreciation and enjoyment of them.

Despite the growing body of research of arts in dementia care, there also remains an ongoing debate around their efficacy. Mirabella (2015) argued there have been few rigorous clinical investigations, resulting in little robust empirical evidence to allow for a full assessment of the advantages and disadvantages of participation in art interventions. The interventions themselves are often heterogenous, poorly defined, and the methods used to evaluate efficacy are varied and often lack rigour. In health service settings, empirical evidence from robust randomised controlled trials (RCTs) of visual art is vital if the potential benefits of such interventions are to be adequately understood and the approach is to be incorporated into 
everyday care. However, undertaking an RCT is challenging, and a number of uncertainties need to be clarified before proceeding to a full-scale trial. Thus, feasibility testing is an essential stage of developing and implementing complex interventions (Medical Research Council, 2006), and is considered best practice to conduct prior to investing time and resources into a full-scale study (Craig et al., 2008). Therefore, the purpose of the present study was to address issues of feasibility in delivering a newly developed, person-centred, therapeutic visual art intervention that is clearly defined and replicable in future research. These include:

(1) Was the intervention practicable and acceptable to the participants and facilitators?

(2) Was the intervention feasible to deliver? Were the processes and features of the intervention feasible?

(3) What were the participants' perceptions of the intervention? Was there evidence of positive effects on the measures of participants' QoL, social functioning and activities of daily living (ADL)?

\section{Methods}

\section{Ethical Approval}

Ethical approval was granted by [blinded for peer-review]. All study participants provided written informed consent to take part.

\section{Intervention Description}

The person-centred, therapeutic visual art intervention is an evidence-based programme that is delivered to people with dementia. The intervention manual was modelled on the successful components of existing visual art interventions [blinded for peer-review], alongside qualitative interviews with the target population and visual art professionals [blinded for peer-review]. Prior to implementation, informal consultations were conducted with the facilitators delivering the sessions to provide practical input on revisions prior to implementation.

The aim was to recruit a maximum of six people with dementia per group. This parameter was applied based on data obtained from the qualitative interviews, where the majority of participants noted that smaller groups are beneficial as they decrease the chance of confusion 
and anxiety compared to a larger group, and facilitate a cohesive group process [blinded for peer-review]. The intervention is comprised of six weekly, one-hour sessions, co-facilitated by two trained facilitators. Sessions were held in a spacious room at a day care centre and at an assisted living facility.

The intervention consists of structured, person-centred session plans, as the activities are strengths-based (focusing on internal strengths rather than the weaknesses or shortcomings associated with dementia) and process-orientated (focusing on the creative process), rather than product or performance oriented (focusing on creating an aesthetically pleasing product). Each week there is a different activity inspired by a different theme and involves a variety of materials (e.g. paints, ink, pencils, fabric, 3D materials) and techniques (e.g. brush, paint roller, pipettes) aimed towards stimulating participant choice and independence. The session structure involved: introductions and warm-up exercises (5-10 minutes), the artistic activity (40minutes), and a debriefing exercise (5-10 minutes). The warm-up exercises aimed to help participants to relax before engaging in the artistic process, and encourage a more immediate involvement. The debriefing exercise allows participants to reflect on their experience, discuss and express their thoughts and emotions relating to the intervention in a confidential way, and continues to develop social networks within the group.

Prior to the six-sessions, an informal 'getting to know you' session is delivered, designed to gain insight into individual's artistic preferences and what they wish to achieve from attendance. The facilitators and participants work together to identify individualised goals prior to the commencement of the first session (e.g. learning a new artistic technique). The facilitators review these goals for each participant mid-intervention to ensure the following sessions would assist participants in achieving their goal. If the facilitator believes the goals are not being met, action plans would be put in place. The subsequent session plans are outlined in Figure 1. The full manual may be available on request from the first author.

[Insert Figure 1]

\section{Participant Recruitment}

The participants were recruited from one day care centre and one assisted living facility, both located in Northern England. Feasibility studies are not expected to have large sample sizes 
that are needed to adequately power statistical null hypothesis testing (Tickle-Degnen, 2013). Previous feasibility studies of creative interventions (including music, drama, dance and visual arts) were explored to determine an appropriate sample size, due to the restricted evidence that exists for visual art feasibility studies alone. Sample sizes for studies involving a range of art forms frequently used small sample sizes, ranging from four to eighteen people with dementia (e.g. Hsu, Flowerdew, Parker, Fachner, \& Odell-Miller, 2015; Loizeau, Kündig, \& Oppikofer, 2015; Low et al., 2016).

\section{Visual art facilitators}

Facilitators were approached after participation in the qualitative interviews during development and expressed an interest in intervention delivery. The first author contacted the facilitators via telephone and performed an eligibility check to confirm each facilitator met the inclusion criteria: (1) a minimum of five years' experience delivering visual art sessions; (2) a minimum of three years' experience delivering visual art sessions to people with dementia, and (3) have up-to-date training in the person-centred approach within dementia care, aligning with their organisational requirements. The facilitators would be excluded if they were a Health and Care Professions Council registered art therapist, or training to become one.

\section{Participants with dementia}

Recruitment began by liaising with a senior staff member at each site to identify potentially eligible participants. Eligibility requirements included: (1) a diagnosis of dementia, a Functional Assessment Staging Tool (FAST) score between 4 (mild dementia) and 6d (moderately severe dementia); (2) the ability to participate in a visual art session (e.g. able to hold and use materials/equipment), and (3) the ability to communicate in English. Participants were excluded if they had attended a person-centred, therapeutic visual art intervention in the past, so they were not influenced by a previous experience for the purpose of the research aims.

Once all eligible participants were identified, an initial assessment of capacity was undertaken by the senior staff member in accordance with the Mental Capacity Act (2005). If the participant was deemed to have capacity, written informed consent was obtained from them prior to the intervention commencing. Where participants were assessed as lacking capacity, a 
personal (relative or friend) or nominated (staff member independent of research) consultee was appointed to provide advice on their wishes.

Participant Demographics

Fifteen people participated in the two groups; four visual art facilitators and eleven people with dementia. At the day care centre, two visual art facilitators delivered the intervention to a group of five participants with dementia. At the assisted living facility, two visual art facilitators delivered the intervention to a group of six participants with dementia (see Table 1).

[Insert Table 1]

\section{Design and outcome measures}

This study was a mixed-methods, quasi-experimental, pre/post design. Quasi-experimental designs aim to evaluate interventions but do not use randomisation. We assessed the impact of the intervention on QoL, ADL and social functioning at three timepoints (T0: baseline, T2: post-intervention, T3: one-month follow-up). Outcome measures were rated on established test instruments. The first author administered the measures with the participants with dementia.

To assess QoL, the Quality of Life in Alzheimer's Disease (QoL-AD; Logsdon, Gibbons, McCurry, \& Teri, 1999) was administered. The QoL-AD has good internal consistency, validity and reliability (Logsdon et al., 1999). Furthermore, the European consensus on outcome measures for psychosocial interventions in dementia (Moniz-Cook et al., 2008) recommends the use of the QoL-AD, as it is brief and has demonstrated sensitivity to psychosocial interventions (Orrell et al., 2014). To assess social functioning, the Social Functioning in Dementia Scale (SF-DEM; Sommerlad, Singleton, Jones, Banerjee, \& Livingston, 2017) was administered. The SF-DEM has content validity and concurrent validity (Sommerlad et al., 2017). It is a reliable and acceptable measure of social functioning of a person with dementia of any severity (Budgett et al., 2019). The Lawton Scale of Activities of Daily Living (Lawton \& Brody, 1969) was administered to assess ADL. This measure has high test-retest and concurrent validity and is consistent with other measures of temporal decline in people with dementia (Johnson, Barion, Rademaker, Rehkemper, \& Weintraub, 2004). The baseline data collection took place a few days before the first session, and the post-intervention 
data was collected within a few days after the last session. Data was collected from $100 \%$ of the participants.

Qualitative interviews were conducted mid-intervention (T1: within the third week), and at T2, based on a topic guide developed by the research team. Interviews were used to explore participant opinions regarding intervention feasibility, facilitators and barriers, and the perceived impacts. All participants completed interviews at the two timepoints.

\section{Data analysis}

The Statistical Package for Social Sciences, version 22.0 (IBM®, 2012), was used for statistical analyses. The data was normally distributed, so a one-way ANOVA was used to determine whether there was a difference in the mean scores on outcome measures between timepoints T0, T2 and T3. To ascertain the direction of effect, a Bonferroni correction was performed on the data.

Data obtained from the interviews were analysed using both inductive and deductive thematic analysis, incorporating the data-driven approach of Boyatzis (1998) and the use of an a priori code template outlined by Crabtree and Miller (1999). The a priori template was based on the research aims, interview questions and evidence derived from the developmental stages. Five broad code categories formed the code template: (1) acceptability and feasibility; (2) group work; (3) participant choice; (4) visual art content, and (5) perceived impact. Data were analysed independently by the first author and then consensus was reached by agreement with the second and third author.

\section{Results}

\section{Pre-post comparison}

Descriptive statistics for the outcome measures at different timepoints are presented in Table 2 .

[Insert Table 2] 
There was an effect of time on social functioning and QoL, but not ADL. The social functioning score showed a significant increase, $F(2,30)=12.39, p=.000$, as did the QoL score, $F(2,30)$ $=10.40, p=.000$. The Bonferroni correction showed the social functioning and QoL scores differed significantly from $\mathrm{T} 0$ to $\mathrm{T} 2, p=.000$ and $p=.001$, respectively. However, both scores did not differ significantly from T2 to T3, $p=1.00$ (see Figures 2 and 3).

[Insert Figure 2]

[Insert Figure 3]

There was no effect of time on $\operatorname{ADL}, F(2,30)=0.011, p=.989$, and the Bonferroni correction showed the ADL score never differed significantly between timepoints T0, T2 and T3, $p=1.00$ (see Figure 4).

[Insert Figure 4]

\section{Interviews}

Interviews were conducted with four facilitators and eleven people with dementia. The interviews with facilitators were conducted in pairs and the interview durations ranged from 21 minutes 06 seconds to 43 minutes 26 seconds. The interview durations for participants with dementia ranged from 5 minutes 02 seconds to 19 minutes 10 seconds. Five overall themes were identified with a number of sub-themes (see Table 3).

[Insert Table 3]

\section{Feasibility and acceptability}

The facilitators reported that the intervention was acceptable and feasible to deliver, although some modifications were proposed to enhance this. When discussing feasibility and acceptability, the interviews focused on elements that are encompassed as sub-themes.

\section{Recruitment}


The facilitators expressed apprehension regarding the recruitment and retention of participants with dementia. They discussed that people may experience trepidation about participating in visual arts, which might influence their receptiveness to the intervention.

It's often those who have never participated and it's that strong fear of creativity [...] the feeling of potential failure, or the advanced artist who is trying to recapture or compare something to what they could do in their earlier life. Because of this I do think it is harder to get people involved and to maintain that engagement. (Facilitator: 001)

The facilitators noted that the fear of creativity eased when participants understood the main focus was on the creative process, rather than producing an aesthetically pleasing product. Creating a non-judgemental, failure-free environment was acknowledged as a key element to recruitment and retention.

When they realised it wasn't an intense high-quality art class, it was about creating something to reflect themselves, express themselves, have fun, that's what got them, and that's what kept them. (Facilitator: 004)

\section{Frequency and duration}

The participants believed that 60-minutes was an appropriate and feasible duration, including 10-minutes for a beginning and an end. It was frequently mentioned that if the sessions were longer, they may be too tiring for the person with dementia, or they may start to disengage. Conversely, if they were shorter, there would not be sufficient time for the participant to engage with the artwork.

It slotted into my day nicely and was a good amount of time. (PwD: 001)

Any longer would probably be too long and any shorter we probably wouldn't be given enough time. So, the time at the beginning is perfect for everyone to get their ideas and have a chat and get settled [...] and then a good solid 40-minutes of art-making, and then gives that time for the discussion at the end. (Facilitator: 003)

The facilitators agreed that completing one weekly session for six-weeks was feasible, and a longer duration may not be appropriate due to factors such as motivation, mood or needing to 
prioritise other tasks. One facilitator commented that a weekly session is sufficient, as participants should not feel pressurised to complete multiple sessions a week as failing to reach this target may de-motivate them.

I think you'd find it very difficult if it was more sessions a week or any longer, I think we'd find it difficult if we didn't achieve it, as would the residents to a degree, because there would be that sense of failure. (Facilitator: 002)

\section{Facilitator skill set}

The facilitators believed they were capable of implementation and could offer training/support to others who delivered the intervention in the future. They reported key skills were: dementia knowledge, experience with visual art and understanding the person-centred approach.

I think the facilitator's do need a particular skill set of dementia knowledge [...] knowledge of dementia, knowledge of each other, and I guess knowledge of the materials and art as well. (Facilitator: 001)

\section{Group format}

Delivering the sessions to a small group was highlighted as feasible, acceptable and would likely lead to enhanced socialisation. The facilitators noted that a maximum of six was an optimal group amount.

I like that it's a smaller group, I like getting to know people a bit more who come here. (PwD: 003)

Smaller groups are great for the intimate socialisation and discussion of ideas, enough for us to be able to facilitate effectively, too. Any more than six and it would be a little too big. (Facilitator: 004)

\section{Practical challenges}


One facilitator identified practical issues that may arise whilst delivering the intervention in an assisted living environment. She highlighted potential challenges around appropriate room availability.

It would be hard to book out a room [...] it would be away from each of the houses, so we would have to get each person to arrive and they may not all arrive at the same time, so we could have people sitting there at the beginning and having to wait, getting bored and losing that engagement before we've even started. (Facilitator: 001)

The facilitator also highlighted that some care staff did not appreciate the intervention structure, and that it was designed for a small, consistent group. Care staff would often interrupt the session to ask the facilitator whether other residents could join.

I think what you're always going to get in this environment is that, 'can we give you this person?', and that's not really okay, because actually we're already working with six residents, so you've got six less people for you to be working with now. (Facilitator: 001)

Thus, it appears important that additional information regarding the intervention is required for the staff team, so they have a greater awareness of what the sessions entail, the group capacity, the protected nature of the group, and the overall purpose of what the facilitators are trying to achieve. Lastly, one facilitator suggested it may be beneficial to have a volunteer in the session to ensure the facilitators remained focused on the creative process.

Maybe having a volunteer [...] so it doesn't result in missed opportunities or taking away from the artistic process. Like helping these situations, so P8 wanted her glasses and she got up and it took a while to sit back down and she could have fallen, and in that time for her, that wasn't the art group anymore. (Facilitator: 001)

\section{Participant choice and autonomy}

Material selection and creating own ideas 
The facilitators felt the intervention was beneficial as it catered to individual preferences/needs, whilst the delivery remained standardised across two sites.

I think what is key to the engagement of everyone, [...] is it has the structure and can be replicated across different places, but people can come in, pick what they want to do and run with it. (Facilitator: 001)

It's wonderfully person-centred. Doing it this way really ensures that the participants really lead the session, making it their own completely and we are really following them. (Facilitator: 001)

Participants with dementia discussed how they valued their independence and appeared to feel empowered as a result of creating their own ideas and selecting their own materials.

I come to each group thinking 'I wonder what we are doing next!' Yes, the choice is marvellous, learning new skills is something you don't expect to do anymore. (PwD: 002)

Participants expressed frustration when they were not able to express their own ideas in previous groups, suggesting participant choice was a key element to successful implementation.

I have come to groups before and I have been set a task and it is often dismissed whether I like it or not. (PwD: 001)

Implementing participant choice

No facilitators identified any barriers for implementing participant choice. They acknowledged the importance of knowing each participant to aid participant choice, highlighting the benefits of the 'getting to know you' session that was delivered prior to the six-sessions.

The key is knowing the person; we need to understand what they like and what they don't like. I think that's why the session before the first one was so advantageous for us both, I mean, both of us and the person with dementia. (Facilitator: 004) 
One facilitator acknowledged that someone with advanced dementia may become overwhelmed by the presence of a variety of materials. She suggested if someone was to become overwhelmed, participant choice is still feasible in different ways.

I think sometimes we can overwhelm people - especially if they are more advanced [...] I think choice can be through what colours [...], he's already picked pastels so now the choice lies within what coloured pastels and what he wants to do with those pastels. (Facilitator: 001)

\section{Socialisation and the group setting}

All participants identified the benefits of the group format, highlighting that this mode of delivery enhanced social functioning and offered opportunities for a sharing experience.

\section{Enhanced social functioning}

There were common threads in the data for both facilitators and people with dementia that related to social functioning, including the opportunity for people with dementia to strengthen relationships, create new ones and discuss ideas.

We had a chat because she was struggling to think of an idea, and it was just nice to have that opportunity to discuss ideas that wouldn't have been possible if she wasn't there. (PwD: 002)

I am surprised because there are people in there who don't normally talk to anyone, or they are often reluctant to at least. It's great to see so many of them chatting like I've never seen before. (Facilitator: 003)

Facilitators regularly highlighted that the consistent group setting with familiar faces and frequent socialisation led to a sense of belonging that maybe was not present before. 
We've got this world that they've got something within. They've got this humour running with both of us, that sense of belonging to a group that's just wonderful. (Facilitator: 001)

\section{Sharing experience}

Participants with dementia would often share their artwork with each other, and frequently praise one another's work.

There was a lovely kind of critique almost at the end, so that was a lovely social moment. That was a lovely moment, a group of people who live together talking about their artwork. (Facilitator: 001)

It's wonderful to look at what other people have done. Everyone has made such beautiful things, it's lovely. (PwD: 004)

The sharing experience was not restricted to within-sessions, as one participant talked frequently about how she shared her artwork with her daughter after each session, an experience she clearly valued.

I drew a pink house last time I was here, and my daughter said she was proud of me. (PwD: 006)

The facilitators at this site also referred to this sharing experience, suggesting it was equally valued by the family member.

Because her daughter was saying that she didn't really do things like that, so for her to talk about it [...], it's just lovely. I think her daughter was really surprised she had remembered that. (Facilitator: 002)

\section{Mentally stimulating activities}

Stimulating and enjoyable activities 
People with dementia suggested that the activities provided were valuable for both mental and physical stimulation.

They're very, very good, because not only do they make you think - you're thinking, you're using your fingers, cutting out and that is very, very important - to use your fingers - because you've heard of the saying, "use it, or lose it! (PwD: 002)

Participants with dementia spoke about mentally stimulating activities as a way of occupying their time in a meaningful way, linking being active to retaining a sense of purpose.

These kinds of activities are good to get me out the house, they mean something and get me doing something different and makes me remember I can do more, I do have purpose, I can do these things. (PwD: 003)

The facilitators noted benefits of mentally stimulating activities; including improvements in mood, motivation and helping the person to think more clearly. There was consensus that it was not important whether the participant could remember the activity, as long as they had enjoyed it during the session and had felt stimulated during that hour.

The activities we have delivered have definitely been mentally stimulating for them, made them think more, thinking about what they want to create and what they want to do. They have that opportunity to be independent and create something for themselveshas increased their motivation and massively improved their mood. (Facilitator: 004)

\section{Activities and themes}

All facilitators suggested the range of activities and themes stimulated the mind, were enjoyable and provided opportunities to learn new skills.

They've been appropriate for people with dementia, for a range of skills, allowing for a range of mentally stimulating activities whilst sticking to a structured theme. (Facilitator: 004) 
The facilitators also suggested that the check-in activities were mentally stimulating, encouraging each individual to think about the theme, what they wanted to produce and have the opportunity to discuss their thoughts and ideas with each other.

I think the warm-up activities; the conversation starters have worked particularly well. I think sometimes when we go in there [...], "we are going to do this, we are going to do that", but you need that 10 minutes at the beginning where you just sit and talk and do something to get you thinking. " (Facilitator: 002)

\section{Perceived impact on participants}

Sense of self/identity

The facilitators highlighted that creativity allowed participants to express themselves, and reconnected people to their sense of identity.

A safe and supportive environment where one can be creative to express themselves, where we focus on what they can still do and what they enjoy to do, rather than what they can't do, acknowledging their contributions and respecting them. That contributes in great ways to their sense of identity, feeling valued, making sure that they don't feel isolated or forgotten. (Facilitator: 001)

One participant with dementia mentioned that attendance had given her a sense of control and a feeling of confidence as a result of being able to achieve or create something. This appeared to be linked to her sense of self and identity:

I started coming to groups here because it gave me something to do, but this has really made me think and realise I can do different things, I can do it! It's given me a sense of just being me again. (PwD: 002)

Quality of life 
Participants with dementia frequently referred to living well in the context of enjoying the fun sessions, being part of a wider social group, and having the opportunity to focus on their strengths rather than deficits often associated with dementia.

The absolute joy I felt coming here, I was able to put any worries aside for the moment and just enjoy the beautiful art everyone made. (PwD: 004)

The facilitators felt the intervention had been empowering for the participants, allowing them to take control, and experience a meaningful activity. One facilitator stated that meaningfulness was synonymous with life quality, acknowledging that meaningful engagement was critical with many opportunities to be 'in the moment'.

For that period of time it definitely does increase people's quality of life [...] something that means something to them, it captures their uniqueness which is something that should always be treasured and protected. Engaging with that kind of opportunity is what makes for higher quality of life because you can be yourself just in the moment. (Facilitator: 001)

\section{Confidence and self-esteem}

There was a common thread throughout the data that participants with dementia enjoyed producing their own artwork, as this led to a feeling of success which was valuable in terms of personal satisfaction and a sense of achievement. Subsequently, this boosted their confidence and self-esteem.

A sense of confidence at the end of the session, purely through the act of creating something, and knowing they can now achieve it. (Facilitator: 002)

Most people with dementia stated they had no previous artistic experience. Participants highlighted how this had given them the opportunity to learn a new skill and subsequently enhanced their confidence and self-esteem. 
Well I'd never done art before, this is all absolutely brand new to me, I'd never done anything like this before. Never, never done any painting [...] I am so proud of myself; I am really proud. (PwD: 002)

\section{Discussion}

This study has demonstrated the acceptability and feasibility of the newly developed visual art intervention, as well as tentatively indicating enhanced social functioning and QoL scores for people with dementia. The feasibility of implementation may be underpinned by a number of factors. Firstly, the rigorous development process of the intervention and its manualised format ensured the sessions were appropriately tailored and fit for purpose. As part of the developmental process, the facilitators participated in informal consultations to provide feedback on the manual prior to delivery. Research has argued that involving the facilitators in the developmental process promotes good decision-making on the selection, planning and implementation of acceptable interventions (Bartholomew, Parcel, Kok, Gottlieb, \& Fernández, 2011). Additionally, the standardised manual and description of elements/processes will support future replication and evaluation. Secondly, the successful implementation may be attributable to the expertise of the facilitators who had good knowledge of artistic practice and were well-trained and knowledgeable about the impact of living with dementia. Consistent with previous literature, these elements may be fundamental to the success of a visual art intervention (Windle et al., 2017).

Thirdly, the frequency and duration may have contributed to the successful implementation by providing weekly one-hour sessions over a six-week period. Beneficial patterns have been observed in interventions with an art-making component with a duration of one-hour (e.g. MacPherson, Bird, Anderson, Davis, \& Blair, 2009; Sauer et al., 2016). In addition, previous literature has suggested a longer intervention duration may be a barrier to regular attendance (e.g. Rusted, Sheppard, \& Waller, 2006). This echoes the findings in this study, as facilitators suggested participants with dementia may become demotivated if the intervention duration is too long.

Lastly, the structure and content of the intervention was experienced to be feasible, appropriate and meaningful. The programme focused on creativity rather than memory or recall, which can often be distressing for people experiencing cognitive impairment (Bohlmeijer, Roemer, 
Cuijpers, \& Smit, 2007). It was emphasised that the artistic activities were enjoyable, gave a sense of control and achievement, and involved a wider social group. In this way, the activities and subsequent accomplishments appeared to have a symbolic function linked to identity, QoL and a sense of belonging, rather than simply being an activity to 'pass time'. Thus, participation and maintained engagement were perhaps due to the meaningful and tailored content of the intervention. Previous visual art interventions that have been designed to be meaningful, failure-free and adopt a primary focus on the creative process as opposed to factual recall have also led to positive outcomes for people with dementia (e.g. Sauer et al., 2016; Windle et al., 2018). Moreover, tailored visual art interventions that participant autonomy and choice reported positive results (Eekelaar et al., 2012; Sauer et al., 2016). Given the personal nature of the visual arts, this is an important but often overlooked consideration in research design. Rather than assign a structured art activity to an individual and assume it will be meaningful to them, the current findings suggest it would be more beneficial to ask participants about their preferences, obtain information about their interests, likes and dislikes, and be able to offer tailored tasks within the intervention.

As the group dynamic appears important for enhanced social functioning, it makes it challenging to ascertain whether the improved scores were solely due to the group setting, rather than a combination of the group and the artistic activity. However, a previous study conducted by Windle et al. (2018) obtained individual perceptions of a visual art intervention. In some cases, the art focus provided the main incentive to attend, whereas for others, the social aspect was more important. However, "a number perceived the benefits of both, making connections between the art and social aspects" (p. 416). Likewise, participants in this study frequently mentioned socialisation and peer support through sharing the visual art activities. This finding suggests the benefits of combining the two elements, and thus indicates the group dynamic should be considered alongside the activities and materials used. This combination may help to facilitate successful uptake and implementation. Furthermore, this indicates the enhanced scores were likely due to a combination of shared activity and social interaction. Thus, conducting a future controlled study, in which a socialisation group activity (excluding arts-based approaches) is used as the control group is warranted.

Although the ADL scores did not improve for any participants, they were maintained across the intervention duration and follow-up. However, the intervention was not designed to target functional abilities, rather, it was intended that enhanced QoL and social functioning may 
motivate participants to engage more actively in ADL. Moreover, the Lawton Scale of Activities of Daily Living (Lawton \& Brody, 1969) used in this study addresses objective capability rather than more subtle factors such as motivation, which might be more likely influenced by a psychosocial intervention. Still, there was no decline in ADL scores and this itself could be considered a positive outcome, although a comparison with a control group in the future would be required to validate this claim.

Overall, this research demonstrates how community stakeholders' engagement and service user involvement serves as an important foundation in improving arts-based interventions for people living with dementia. Such involvement is especially pertinent in visual arts considering this arts-based approach requires further research development (Windle et al., 2017). Moreover, this research highlights that the intervention can be delivered by non-specialist staff, reducing potential cost implications that may have inhibited implementation. This may facilitate successful implementation and sustainability in other care settings. Additionally, the findings indicate that supporting participant choice is a key element, offering participants tailored activities that do not result in monotony or conversely, fear of partaking in creativity. Considering this balance is vital to effective planning and implementation of visual art interventions in a care setting. Lastly, this research recommends that visual art interventions should consider a wide range of participants in attendance, rather than just those who have an existing interest or ability in visual art. The current intervention has an absence of external assessment with regards to existing ability, and focuses on the creative process as opposed to creating an aesthetically pleasing product. This appeared to engage and capture the interest of those with limited abilities, indicating that the benefits of creativity can extend beyond just participants with existing artistic experience.

\section{Limitations}

Although the interview topic guides were designed to explore intervention strengths and challenges, there did appear to be an over-representation of positive perceptions. Whilst this could be reflective of the reality, it is also plausible that the findings may have been influenced by a social desirability bias. Secondly, given this was an acceptability and feasibility study, the study was not adequately powered and there was no control group which restricts what can be extrapolated regarding efficacy, and means the results must be interpreted with caution. Future 
evaluation of the intervention is required that incorporates randomisation with a control condition.

\section{Conclusion}

This feasibility study has demonstrated implementation of the visual art intervention was feasible and acceptable to both facilitators and people with dementia, whilst highlighting which factors are perceived as important. Engagement with visual art represents a holistic yet individualised intervention that can be difficult to study systematically. By utilising a mixedmethods research design, this paper used strengths from both qualitative and quantitative methods to describe a multifaceted and complex picture of the enriching experience of artistic engagement. Consequently, taking into account the factors that impact on feasibility and acceptability will promote future robust evaluation through RCT designs.

\section{Disclosure of Interest}

The authors report no conflict of interest. 


\section{References}

Bartholomew, L. K., Parcel, G. S., Kok, G., Gottlieb, N. H., \& Fernández, M. E. (2011). Planning health promotion programs: An intervention mapping approach ( $3^{\text {rd }} \mathrm{ed}$.) San Francisco, CA: Jossey-Bass.

Bohlmeijer, E., Roemer, M., Cuijpers, P., \& Smit, F. (2007). The effects of reminiscence on psychological well-being in older adults: A meta-analysis. Ageing and Mental Health, 11(3), $291-300$.

Boyatzis, R. (1998). Transforming qualitative information: Thematic analysis and code development. Thousand Oaks, CA: Sage.

Brillantes-Evangelista, G. (2013). An evaluation of visual arts and poetry as therapeutic interventions with abused adolescents. The Arts in Psychotherapy, 40, 1, $71-84$.

Budgett, J., Brown, A., Daley, S., Page, T. E., Banerjee, S., Livingston, G., \& Sommerlad, A. (2019). He social functioning in dementia scale (SF-DEM): Exploratory factor analysis and psychometric properties in mild, moderate and severe dementia. Alzheimer's and Dementia: Diagnosis, Assessment and Disease Monitoring, 11, 45 - 52.

Crabtree, B., \& Miller, W. (1999). A template approach to text analysis: Developing and using codebooks. Newbury Park, CA: Sage.

Craig, P., Dieppe, P., Macintyre, S., Michie, S., Nazareth, I., \& Petticrew, M. (2008). Developing and evaluating complex interventions: the new Medical Research Council guidance. $B M J, 337$.

Creative Health, APPG (2017). All party parliamentary groups on arts. Health and WellBeing (APPG) Inquiry Report, available at: www.artshealthandwellbeing.org.uk/appg-inquiry.

de Medeiros, K., \& Basting, A. (2014). 'Shall I compare thee to a dose of donepezil?': cultural arts interventions in dementia care research. The Gerontologist, 54(3), 344-353.

Department of Health (2005). Mental Capacity Act. London, HSMO.

Hsu, M., Flowerdew, R., Parker, M., Fachner, J., \& Odell-Miller, H. (2015). Individual music therapy for managing neuropsychiatric symptoms for people with dementia and their carers: A cluster randomised controlled feasibility study. BMC Geriatrics, 15.

IBM Corp. (2012). IBM SPSS Statistics for Windows, Version 21.0. Armonk, NY: IBM Corp.

Johnson, N., Barion, A., Rademaker, A., Rehkemper, G., \& Weintraub, S. (2004). The activities of daily living questionnaire: A validation study in patients with dementia. Alzheimer's Disease and Associated Disorders, 18(4), 223 - 230.

Lawton, M. P., \& Brody, E. M. (1969). Assessment of older people: Self-maintaining and instrumental activities of daily living. Gerontologist, 9(3), 179-186. 
Logsdon, R. G., Gibbons, L. E., McCurry, S. M., \& Teri, L. (1999). Quality of life in Alzheimer's disease: Patient and caregiver reports. Journal of Mental Health and Ageing, 5(1), $21-32$.

Loizeau, A., Kündig, Y., \& Oppikofer, S. (2015). 'Awakened Art Stories'- Rediscovering pictures by persons living with dementia utilising TimeSlips: A pilot study. Geriatric Mental Health Care, 3(2), 13 - 20.

Low, L. F., Carroll, S., Merom, D., Baker, J. R., Kochan, N., Moran, F., \& Brodaty, H. (2016). We think you can dance! A pilot randomised controlled trial of dance for nursing home residents with moderate to severe dementia. Complementary Therapies in Medicine, $29,42-44$.

MacPherson, S., Bird, M., Anderson, K., Davis, T., \& Blair, A. (2009). An art gallery access programme for people with dementia: 'You do it for the moment'. Aging and Mental Health, 13(5), $744-752$.

Mangione, G. (2018). The art and nature of health: A study of therapeutic practice in museums. Sociology of Health and Illness, 40(2), 283 - 296.

Medical Research Council (2006). Developing and evaluating complex interventions. London, MRC.

Mirabella, G. (2015). Is art therapy a reliable tool for rehabilitating people suffering from brain/mental diseases? The Journal of Alternative and Complementary Medicine, 21(4), 196-199.

Moniz-Cook, E., Vernooij-Dassen, M., Woods, R., Verhey, F., Chattat, R., De Vugt, M., .. . Orrell, M. (2008). A European consensus on outcome measures for psychosocial intervention research in dementia care. Ageing and Mental Health, 12(1), 14 - 29.

Orrell, M., Aguirre, E., Spector, A., Hoare, Z., Streater, A., Woods, B., . . Russell, I. (2014). Maintenance cognitive stimulation therapy (CST) for dementia: A single-blind, multicentre, randomised controlled trial of maintenance CST vs. CST for dementia. BMJ, 204(6), $454-461$.

Palmiero, M., Di Giacomo, D., \& Passafiume, D. (2012). Creativity and dementia: A review. Cognitive Processing, 13, 193 - 209.

Richards, A., Tietyen, A. C., Jicha, G. A., Bardach, S. H., Schmitt, F. A., Fardo, D. W., . . . Abner, E. L. (2018). Visual arts education improves self-esteem for persons with dementia and reduces caregiver burden: A randomised controlled trial. Dementia, 18(78), 3130 - 3142.

Rusted, J., Sheppard, L., \& Waller, D. (2006). A multi-centre randomized control group trial on the use of art therapy for older people with dementia. Group Analysis, 39(4), 517536.

Sauer, P. E., Fopma-Loy, J., Kinney, J. M., \& Lokon, E. (2016). 'It makes me feel like myself': Person-centered versus traditional visual arts activities for people with dementia. Dementia, 15(5), 895-912.

Schall, A., Tesky, V. A., Adams, A-K., \& Pantel, J. (2018). Art museum-based intervention to promote emotional well-being and improve quality of life in people with dementia: The ARTEMIS project. Dementia, 17(6), 728 - 743.

Tickle-Degnen, L. (2013). Nuts and bolts of conducting feasibility studies. The American Journal of Occupational Therapy, 67(2), 171 - 176.

Sommerlad, A., Singleton, D., Jones, R., Banerjee, S., \& Livingston, G. (2017). Development of an instrument to assess social functioning in dementia: The social functioning in dementia scale (SF-DEM). Alzheimer's and Dementia: Diagnosis, Assessment and Disease Monitoring, 7, 88 - 98.

Windle, G., Joling, K., Howson-Griffiths, T., Woods, B., Jones, C., van de Ven, P., . . . Parkinson, C. (2018). The impact of a visual arts program on quality of life, 
communication, and well-being of people with dementia: A mixed methods longitudinal investigation. International Psychogeriatrics, 30(3), 409 - 423.

Windle, G., Gregory, S., Howson-Griffiths, T., Newman, A., O’Brien, D., \& Goulding, A. (2017). Exploring the theoretical foundations of visual art programmes for people living with dementia. Dementia, 17(6), $702-727$.

Zeilig, H., Killick, J., \& Fox, C. (2014). The participative arts for people living with a dementia: A critical review. International Journal of Ageing and Later Life, 9(1), 7 - 34.

(1) Introduction to Visual Arts: A time for participants to be able to 'play around' with materials and techniques and decide what works best for them.

(2) Festival Themes: Each individual can focus on their own preference of season, holiday or festival. This theme is low in complexity and does not impose a significant challenge at the beginning of the intervention.

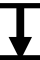

(3) Exploring Emotions: Encourages self-expression through visual arts.

\section{1}

(4) Working with Memories: Builds on the concept of creative reminiscence, providing an opportunity to interact with the art for recreating memories and telling their stories.

(5) Creating to Music: Encourages participants to engage with visual art influenced by the music they are listening to 'in the moment'.

(6) Coming to a Close: This session aims to ensure the participants have had appropriate closure to their artistic process. The focus of this session is the participants journey through the intervention, and what they have accomplished.

Figure 1: Outline of intervention session plans 
Table 1: Demographics of people with dementia and visual art facilitators

\begin{tabular}{|c|c|c|}
\hline \multicolumn{3}{|c|}{ People with dementia (day care centre, $n=5$ ) } \\
\hline Gender & Female & $4(80 \%)$ \\
\hline Mean age (years) & & $84.4(S D=5.8)$ \\
\hline \multirow[t]{4}{*}{ Ethnicity } & White British & $2(40 \%)$ \\
\hline & British Australian & $1(20 \%)$ \\
\hline & Russian & $1(20 \%)$ \\
\hline & Indian & $1(20 \%)$ \\
\hline \multirow[t]{2}{*}{ FAST score } & 4 & $4(80 \%)$ \\
\hline & $6 b$ & $1(20 \%)$ \\
\hline Average length of attendance (years) & & $5.3(S D=55.58)$ \\
\hline \multicolumn{3}{|c|}{ People with dementia (assisted living facility, $n=6$ ) } \\
\hline Gender & Female & $3(50 \%)$ \\
\hline Mean age (years) & & $84.8(S D=5.1)$ \\
\hline Ethnicity & White British & $6(100 \%)$ \\
\hline \multirow[t]{4}{*}{ FAST score } & 4 & $1(16.6 \%)$ \\
\hline & 5 & $1(16.6 \%)$ \\
\hline & $6 a$ & $1(16.6 \%)$ \\
\hline & $6 \mathrm{~d}$ & $3(50 \%)$ \\
\hline Average length of stay (months) & & $11.6(S D=7.05)$ \\
\hline \multicolumn{3}{|l|}{ Facilitators $(n=4)$} \\
\hline Gender & Female & $3(75 \%)$ \\
\hline Mean age (years) & & $44.7(\mathrm{SD}=6.2)$ \\
\hline Ethnicity & White British & $4(100 \%)$ \\
\hline
\end{tabular}


Table 2: Descriptive statistics of outcome measures at baseline, post-intervention and follow-up.

\begin{tabular}{rccc}
\hline \multicolumn{1}{r}{} & T0 & T2 & T3 \\
\hline QoL & & 39 & 38.55 \\
\hline Mean Score & 28.45 & 37 & 38 \\
\hline Standard Deviation & 5.84 & 6.36 & 6.19 \\
\hline ADL & 27 & 2.09 & 2 \\
\hline Mean Score & 2 & 2 & 2 \\
\hline Median Score & 1 & 1.70 & 1.55 \\
\hline Standard Deviation & 1.73 & & 29.09 \\
\hline Mean Score & 16.28 & 30.55 & 26 \\
\hline Median Score & 16 & 29 & 9.20 \\
\hline Standard Deviation & 3.49 & 8.27 & \\
\hline
\end{tabular}




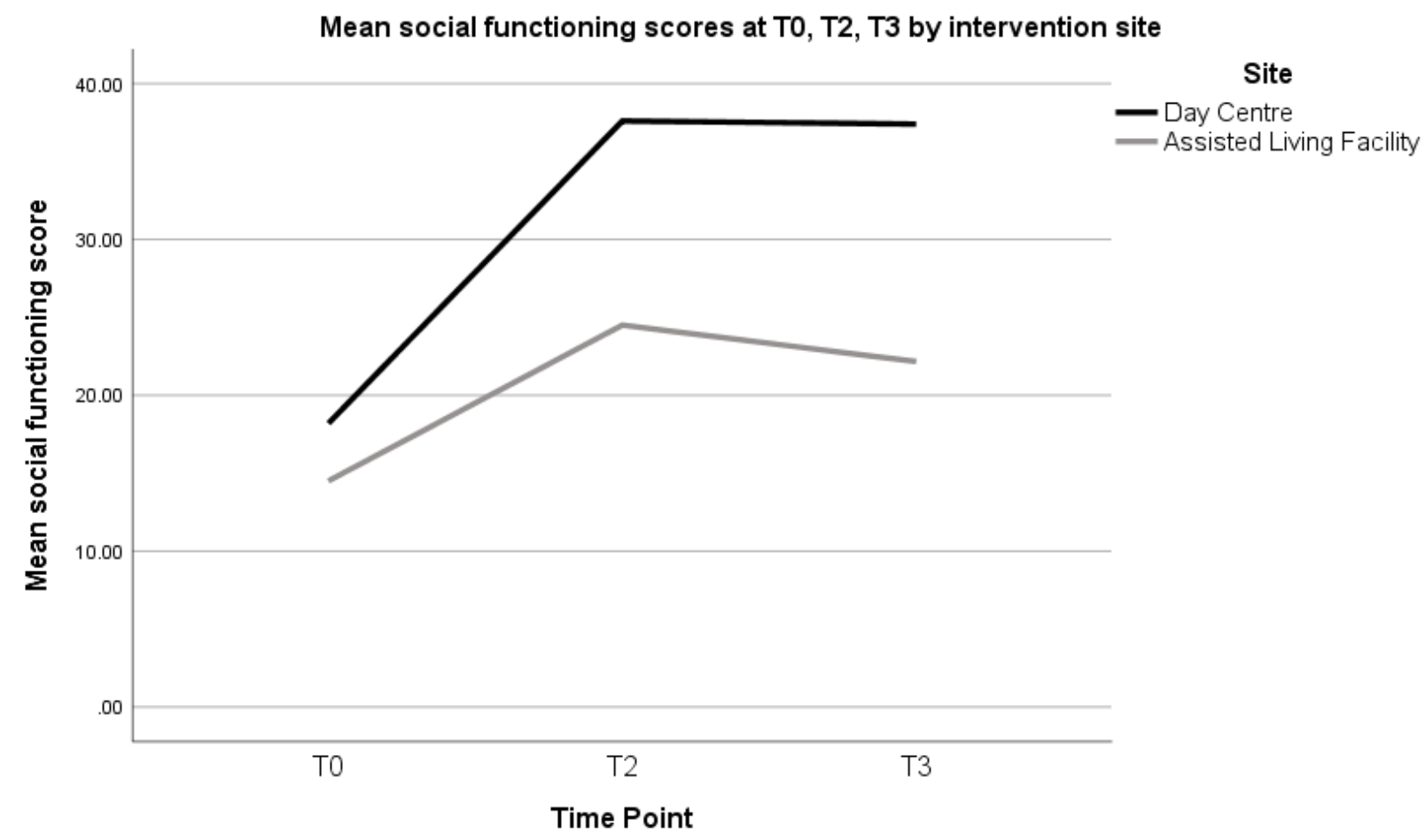

Figure 2: A graph to show the mean social functioning scores at T0, T2 and T3, grouped by intervention site.

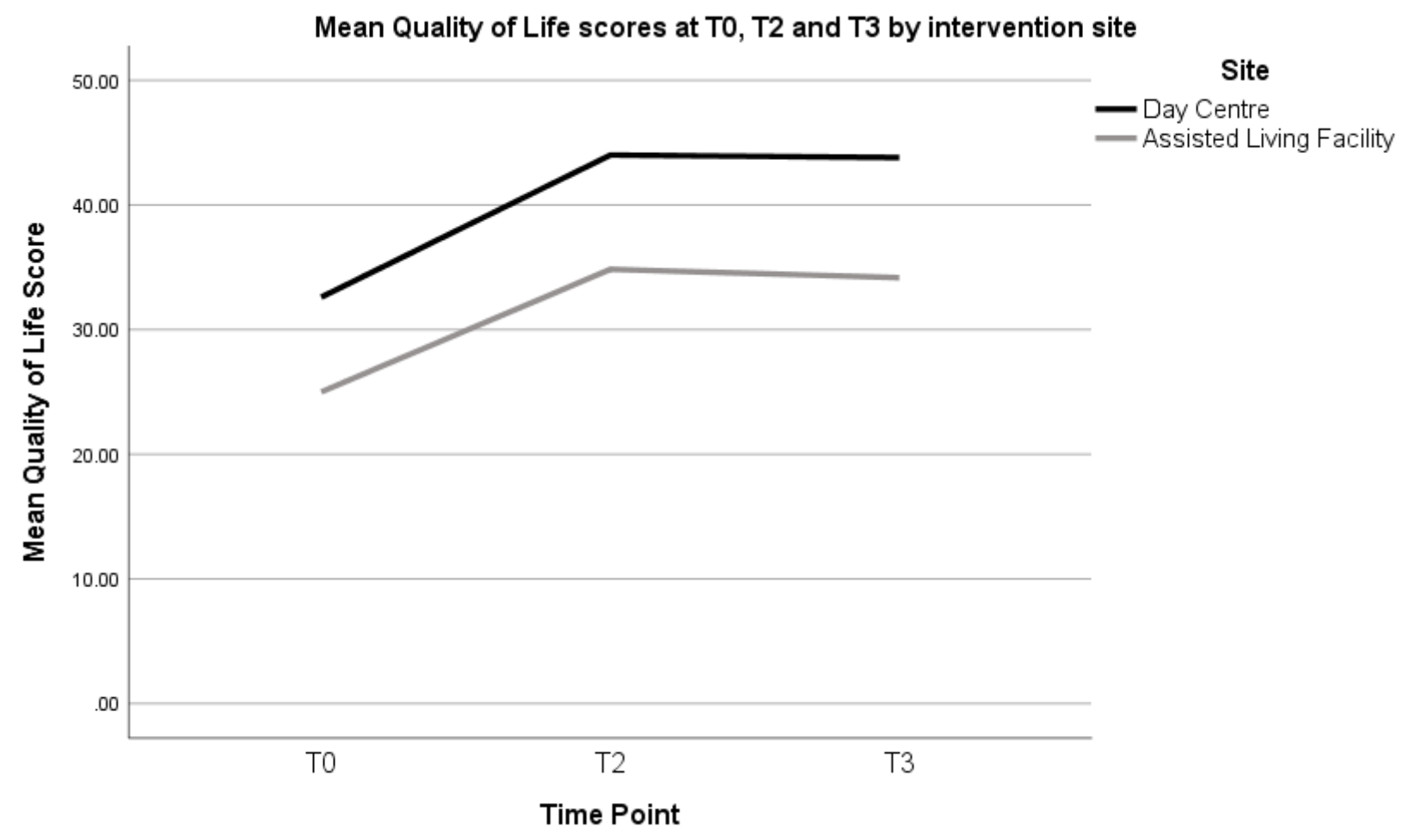

Figure 3: A graph to show the mean quality of life scores at T0, T2 and T3, grouped by intervention site. 


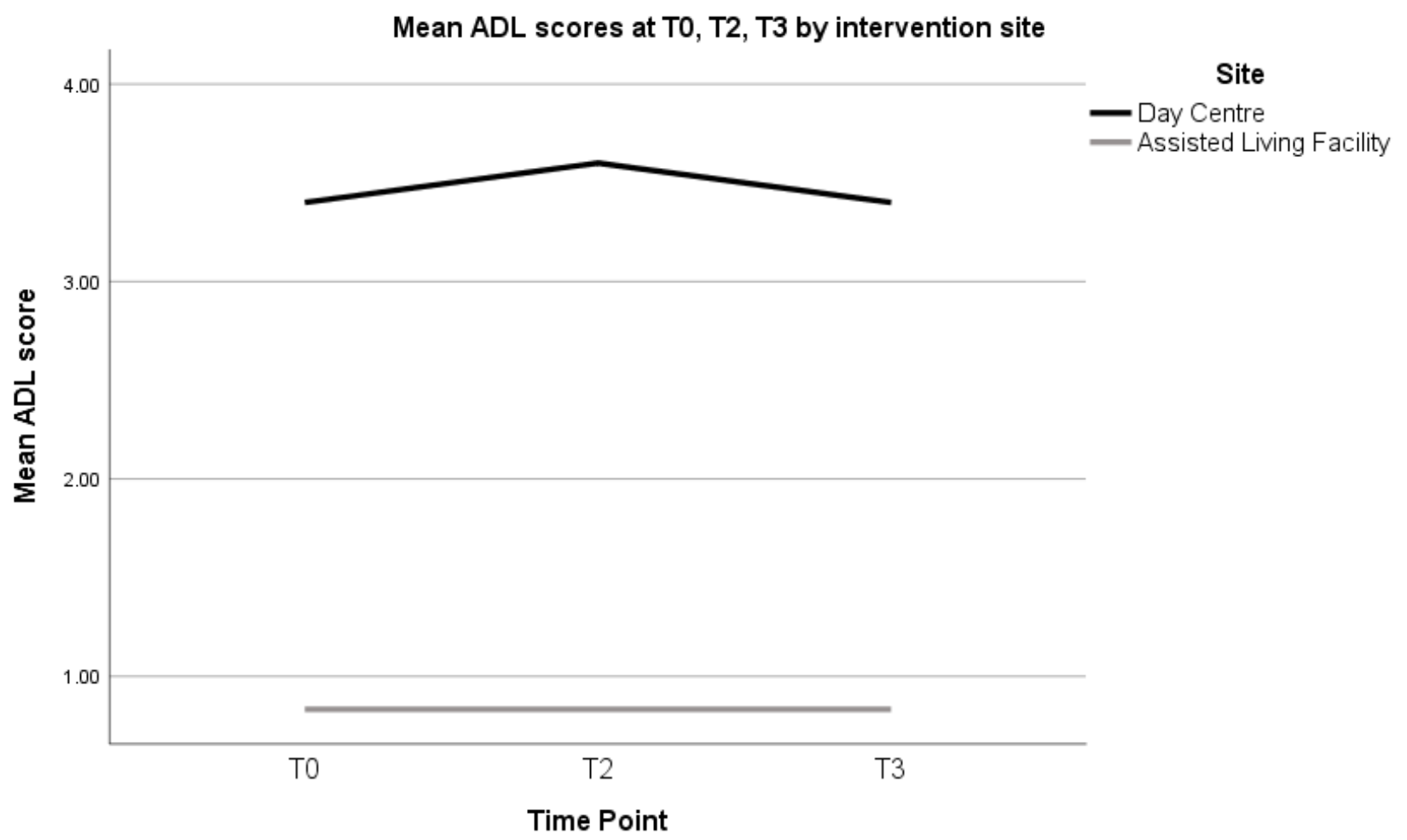

Figure 4: A graph to show the mean ADL scores at T0, T2 and T3, grouped by intervention site.

Table 3: Main themes with a cluster of sub-themes

\begin{tabular}{|c|c|}
\hline Themes & Sub-Themes \\
\hline $\begin{array}{l}\text { Feasibility/acceptability of the } \\
\text { intervention }\end{array}$ & $\begin{array}{ll}\text { - } & \text { Recruitment } \\
\text { - } & \text { Frequency/duration } \\
\text { - } & \text { Facilitator skill set } \\
\text { - } & \text { Group format } \\
\text { - } & \text { Practical challenges }\end{array}$ \\
\hline Participant choice and autonomy & $\begin{array}{l}\text { - Material selection/creating own ideas } \\
\text { - Implementing participant choice }\end{array}$ \\
\hline Socialisation and the group setting & $\begin{array}{l}\text { - Enhanced social functioning } \\
\text { - Sharing experience }\end{array}$ \\
\hline Mentally stimulating activities & $\begin{array}{l}\text { - Stimulating and enjoyable } \\
\text { - Activities and themes }\end{array}$ \\
\hline Perceived impact on participants & $\begin{array}{l}\text { - Sense of self/identity } \\
\text { - } \text { Quality of life } \\
\text { - } \quad \text { Confidence/self-esteem }\end{array}$ \\
\hline
\end{tabular}

